\title{
Die Auswirkungen der SARS-CoV-2-Einschränkungen auf die Patientenversorgung im Fachbereich für Plastische, Rekonstruktive und Ästhetische Chirurgie
}

\section{The impact of SARS-CoV-2 restrictions on medical care in Plastic Surgery}

Autoren

Silvia Bernuth ${ }^{1}$, Raymund E. Horch ${ }^{2}$ O, Adrian Vater ${ }^{1}$, Konrad Fuchs ${ }^{1}$, Michael G. Jakubietz ${ }^{1}$, Karsten Schmidt ${ }^{1}$, Rainer H. Meffert ${ }^{3}$, Rafael G. Jakubietz ${ }^{1}$

Institute

1 Universitätsklinikum Würzburg, Chirurgische Klinik 2, Plastische Chirurgie

2 Universitätsklinikum Erlangen, Plastische und Handchirurgische Klinik

3 Universitätsklinikum Würzburg, Chirurgische Klinik 2, Unfallchirurgie

Schlüsselwörter

COVID-19, Pandemie, Plastische Chirurgie, Einschränkungen

Key words

Plastic surgery, SARS-CoV-2, pandemic, restrictions

eingereicht 01.07 .2020

akzeptiert $\quad 15.07 .2020$

Bibliografie

DOI https://doi.org/10.1055/a-1220-6920

Handchir Mikrochir Plast Chir 2020; 52: 272-279

(c) Georg Thieme Verlag KG Stuttgart · New York

ISSN 0722-1819

Korrespondenzadresse

Silvia Bernuth

Universitätsklinikum Würzburg, Chirurgische Klinik 2, Plas-

tische Chirurgie

Oberdürrbacher Str. 6

97080 Würzburg

Tel.: 0931020137001

Fax: 093120137009

E-Mail: bernuth_s@ukw.de

\section{ZUSAMMENFASSUNG}

Hintergrund Im Rahmen der SARS-CoV-2-Pandemie wurde zur Bewältigung der erwarteten COVID-19-Patienten eine Reduktion der ambulanten und stationären elektiven Patientenbehandlung an den dafür vorgesehenen Kliniken gefordert. Je nach örtlichen Gegebenheiten und Versorgungsauftrag kam es während der SARS-CoV-2-Restriktionen zu unterschiedlichen Auswirkungen auf die Patientenversorgung. Betrachtet werden sollte der Anteil der notwendigen Versorgungsleistungen während der SARS-CoV-2-Restriktionen in einer Plastischen Chirurgie einer Großklinik.

Methode Untersucht wurde die OP-Auslastung einer Abteilung für Plastische Chirurgie einer universitären Klinik im Zeitraum vom 16.03.2020 bis 27.04.2020. Diese Daten wurden mit Daten desselben Zeitraumes der Jahre 2017-2019 verglichen.

Ergebnisse Auf Grund des vollständigen Stopps an elektiven Operationen kam es in dem Zeitraum der Restriktionen zu einer OP-Auslastung von 57,3\% verglichen mit den Jahren zuvor. Das Verhältnis von notfallmäßigen (2020: 56,4\%; 2017-2019: 54,9\%) und dringlichen (2020: 44,6\%; 2017-2019: 45\%) Operationen zu der Gesamtanzahl an OPs zeigte keine deutliche Veränderung. Des Weiteren zeigten sich keine ausschlaggebenden Unterschiede der verhältnismäßigen Verteilung der notfallmäßigen und dringlichen Operationen bezogen auf die Plastisch-Chirurgischen Teilbereiche, die Verletzungsursache oder den Versicherungsstatus (BG/Nicht-BG).

Zusammenfassung Auf Grund der vorliegenden Daten kann die Relevanz des Fachgebiets für die allgemeine Patientenversorgung belegt werden. Daraus ergibt sich eine eindeutige Erforderlichkeit der Vorhaltung von Plastisch-Chirurgischen OP-Kapazitäten und Infrastrukturen auch während Krisenzeiten.

\section{ABSTRACT}

Background To manage the expected COVID-19 patient load major restrictions in in- and outpatient treatment had to be made. Depending on local conditions and order supply differences SARS-CoV-2 restrictions had a massive impact on medical care. To show the impact of plastic surgery on emergency surgery during SARS-CoV-2 pandemic, the amount of surgical emergencies in a single center plastic surgery division were evaluated.

Method The number of plastic surgery cases in a university hospital was evaluated during 16.03.2020 to 27.04.2020 and compared with previous years.

Results Due to cancelling of elective surgery the number of cases dropped to $57,3 \%$ of the caseload of previous years. There was no change in ratio of emergency (2020: 56,4\%; 
2017-2019: 54,9\%) and urgent (2020: 44,6\%; 2017-2019: $45 \%$ ) surgery. No changes in regard to the etiology of trauma cause nor insurance status (occupational insurance/health insurance) were noted.
Conclusion Based on the data of this evaluation there is a clear relevance of Plastic Surgery in the setting of general medical care. Even during the pandemic crises a sufficient plastic surgery service is mandatory in a tertiary referral center.

\section{Einleitung}

Die SARS-CoV-2-Pandemie stellt eine der größten Herausforderungen des 21sten Jahrhunderts dar und brachte weltweit Nationen, unabhängig von ihrem sozioökonomischen Status, an die Grenzen der Belastbarkeit ihrer Gesundheitssysteme [1-3]. Bis Ende Juni 2020 wurden weltweit 9.628 .658 bestätigte COVID-19-Fälle und 489.731 damit assoziierte Todesfälle registriert [4]. Daten weisen darauf hin, dass von diesen Patienten bis zu 7-16\% intensivpflichtig waren $[5,6]$ und zwischen $6-33,1 \%$ eine invasive und nicht-invasive Respiratortherapie benötigten [3, 7]. Die Europäische Gesellschaft für Plastische, Rekonstruktive und Ästhestische Chirurgie (ESPRAS) konnte in einer Übersichtsarbeit zeigen, dass alle befragten europäischen Kliniken im Rahmen der SARS-CoV2-Krise ausschließlich notfallmäßige und dringliche Operationen durchführten und von über zwei Dritteln der Plastisch-Chirurgischen Einrichtungen Mitarbeiter in die Behandlung von COVID19-Patienten involviert waren [8]. Ferner wurden durch die ESPRAS sowie dem Europäischen Verband der Gesellschaften für Ästhetische Chirurgie (EASAPS) Handlungsempfehlungen für die Konsultation von Patienten sowie invasive und nicht-invasive Eingriffe für die Zeit der SARS-CoV-2-Pandemie erarbeitet [9]. Um medizinische Ressourcen, wie Überwachungsbetten, Beatmungsplätze, Fachpersonal und Materialien an Kliniken zu schonen, wurden auf Empfehlung des Bundesgesundheitsministers ab dem 16.03.2020 alle planbaren Operationen, Aufnahmen und Eingriffe auf unbestimmte Zeit verschoben. Ferner sollten alle Kliniken, die in den Krankenhausplan des Freistaates Bayern aufgenommen waren, Universitätsklinika und Krankenhäuser mit einem Versorgungsvertrag nach § 109 des Fünften Sozialgesetzbuches (SGB V) sowie Privatkliniken mit einer Zulassung nach §30 der Gewerbeordnung (GewO), sicherstellen, dass alle zur Verfügung stehenden Kapazitäten für die stationäre Versorgung von COVID-19-Patienten genutzt werden können [10]. Des Weiteren wurde die Akuität der COVID-Entwicklung und die Einordnung der medizinischen Versorgung auf Grundlage der Eskalationseinteilung des American College of Surgeons (ACS) vorgenommen [11].

Phasen der Akuität der COVID-19-Entwicklung des ACS [11]:

- Phase I-semi-akute Phase: wenige COVID-19 Patienten, Krankenhaus-Ressourcen sind nicht ausgeschöpft, freie Beatmungskapazitäten sind vorhanden, keine schnelle Eskalation der COVID-Entwicklung

- Phase II - akute Phase: viele COVID-19-Patienten, ITS- und Beatmungskapazitäten sind limitiert, Verbrauchsmaterialien des OPs sind limitiert oder die COVID-Entwicklung innerhalb der Klinik eskaliert

- Phase III - alle Klinikressourcen werden für COVID-19-Patienten benötigt, keine ITS- oder Beatmungskapazitäten mehr vorhanden, Verbrauchsmaterialien des OPs sind erschöpft
Ab dem 20.03.20 befanden sich alle dazugehörigen Kliniken im Freistaat Bayern vorerst bis zum 15.05.20 in Phase III. Ausschließlich Notfalloperationen waren in diesem Zeitraum erlaubt. Ab dem 27.04.20 wurden diese Restriktionen dahingehend gelockert, dass auf Grund der Diskrepanz zwischen der erwarteten und der eingetretenen Menge an stationär- oder intensivpflichtigen COVID19-Patienten, semielektive Operationen, welche auf Grund von Leidenszuständen, wie beispielsweise Schmerzen, innerhalb von 1-3 Monaten indiziert wären, durchgeführt werden konnten. Dies entsprach der Phase II nach den Empfehlungen der ACS.

Auf dieser Grundlage wurden ab dem 16.03.20 alle elektiven und semielektiven Operationen verschoben und ausschließlich Notfalloperationen durchgeführt. Richtungsweisende Kategorisierungen der Operationsdringlichkeit wurden bereits in der Literatur beschrieben [11, 12]. Somit wurden zunächst in den betroffenen Universitätsklinika allem voraus akute Traumata und Weichteilinfektionen als notfallmäßige Operationen definiert. Ferner wurden als dringliche Operationen nur Weichteilrekonstruktionen im Rahmen von Verbrennungen, Traumata oder Infektionen sowie Tumorerkrankungen eingestuft. Angepasst an örtliche Strukturen konnten tumorbedingte Defekte, insbesondere bei Patienten, die schon vor dem Einsetzen der Restriktionen anbehandelt worden waren, auch während der Phase III durch aufwendige plastisch-chirurgische Rekonstruktionsverfahren versorgt werden. Im Rahmen der Lockerungen der Restriktionsempfehlungen wurden ab dem 27.04.20 schmerzhafte degenerative Indikation und Nervenkompressionssyndrome hinzugezogen.

Das Fachgebiet Plastische und Ästhetische Chirurgie ist anders als mono-organbezogene Fächer wesentlich breiter gefächert und erlaubt daher eine unterschiedliche Gewichtung der 4 Säulen basierend auf den jeweiligen örtlichen Gegebenheiten und dem Versorgungsauftrag. Im Allgemeinen wird das Fachgebiet der Plastischen Chirurgie in der öffentlichen Wahrnehmung meist als hochelektiv angesehen. Somit könnte im Kontext der SARS-CoV-2-Pandemie die Versorgung dieser Patienten als wenig dringlich und nicht erforderlich angesehen werden. Da dies der klinischen Realität widerspricht, sollte in dieser Studie die Dringlichkeit der Indikationen innerhalb des Zeitraums der Pandemie retrospektiv in einem Zentrum mit den Vorjahren verglichen werden, um zu eruieren, wie hoch der rein elektive Anteil der Versorgungsleistung in einer in Großkliniken angesiedelten Plastischen Chirurgie ist. Während eigenständige Kliniken bei der Ressourcenverteilung möglicherweise über eine andere Ausgangsposition bei der OP-Kapazitätszuordnung verfügen, gilt es für den Fall einer organisatorischen Einordnung des Fachgebietes in eine übergeordnete Klinik noch zusätzliche Aspekte zu berücksichtigen. Daher wurde hier ein Vergleich des tatsächlichen OP-Aufkommens in einer integrierten Einrichtung der Maximalversorgung zu den Vorjahren durchgeführt. 


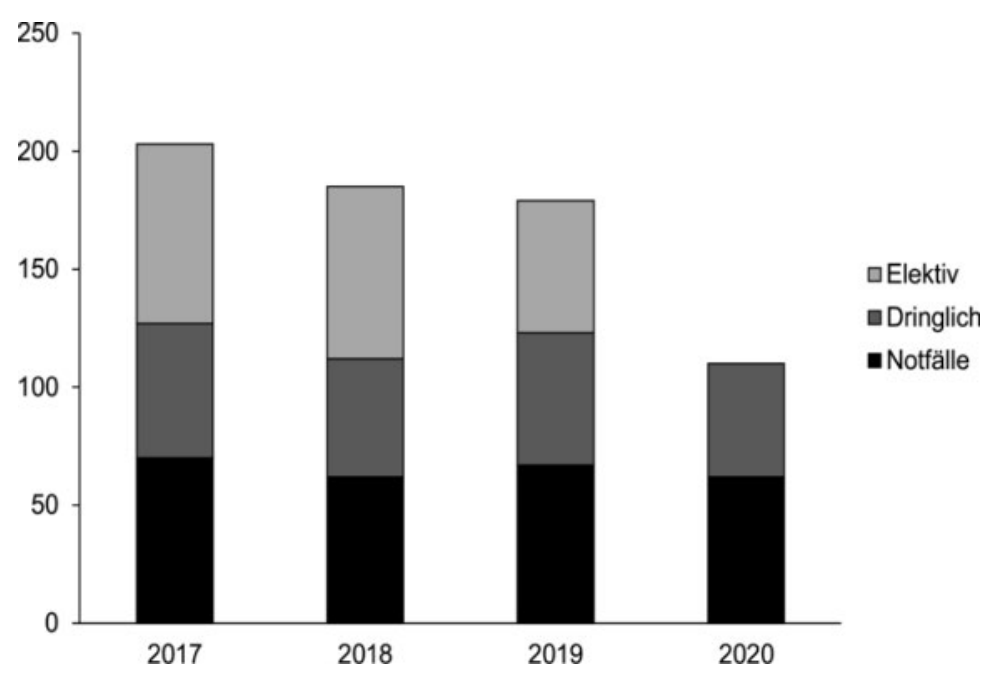

- Abb. 1 Häufigkeiten der durchgeführten Operationen anhand ihrer Dringlichkeit.

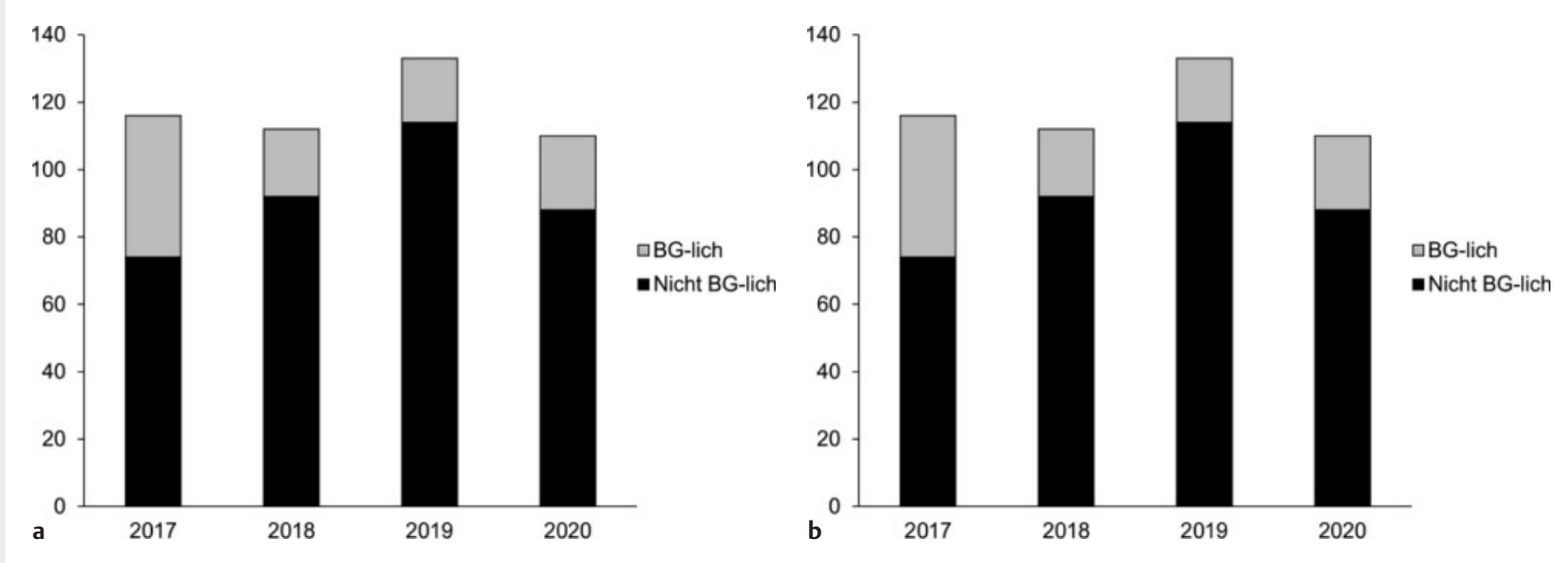

- Abb. 2 a Häufigkeiten der BG-lichen und nicht BG-lich versicherten Fälle inkl. der elektiven Operationen. b Häufigkeiten der BG-lichen und nicht BG-lich versicherten Fälle ohne elektive Operationen.

\section{Methodik}

Beispielhaft erfolgte eine Auswertung der durchgeführten Operationen sowie ein Vergleich des gleichen Zeitraumen der Jahre 2017-2019 in einer Plastischen Chirurgie eines Universitätsklinikums. In diesem Zeitraum galt für das beobachtete Zentrum die Phase-III-Einstufung des ASC, welche ausschließlich die Durchführung von notfallmäßigen Operationen erlaubte. Ferner wurde die Ursache der Verletzungen verglichen, um mögliche Veränderungen des Patientenkollektives zu beurteilen.

Als Notfall wurden Operationen definiert, die innerhalb von $12 \mathrm{~h}$ nach Vorstellung durchgeführt wurden. Hierunter wurden offene Verletzungen der Knochen, Sehnen, Nerven oder Gefäße sowie Infektionen und Erstversorgungen von Verbrennungen zusammengefasst. Operationen innerhalb von 14 Tagen nach Trauma, wie geschlossene Verletzungen der Knochen, Sehnen, Bänder oder Nerven sowie Rekonstruktionen nach Verbrennungen, Trauma,
Infektionen oder Tumorresektionen, wurden als dringliche OP-Indikationen definiert. Diese Kategorie beinhaltete ebenfalls die Entfernung von temporärem, gelenksübergreifendem Osteosynthesematerial. Operationen im Rahmen von Nervenkompressionssyndromen und schmerzhaften degenerativen Erkrankungen wurden als semielektiv eingeteilt. Als elektive Operationen wurden ästhetische Operationen, körperformende Eingriffe, Korrekturen von Fehlbildungen, die Defektdeckung von chronischen Wunden, handchirurgische Eingriffe, wie beispielsweise Artho- und Tenolysen oder Implantatentfernungen sowie Operationen im Rahmen von degenerativen Erkrankungen ohne hohen Leidensdruck definiert.

\section{Ergebnisse}

In den Jahren 2017-2019 wurden in dem 6-wöchigen Zeitraum vom 16.03.20 bis zum 27.04.20 durchschnittlich 192 Operationen durchgeführt, davon durchschnittlich 66,3 notfallmäßig, 54,3 


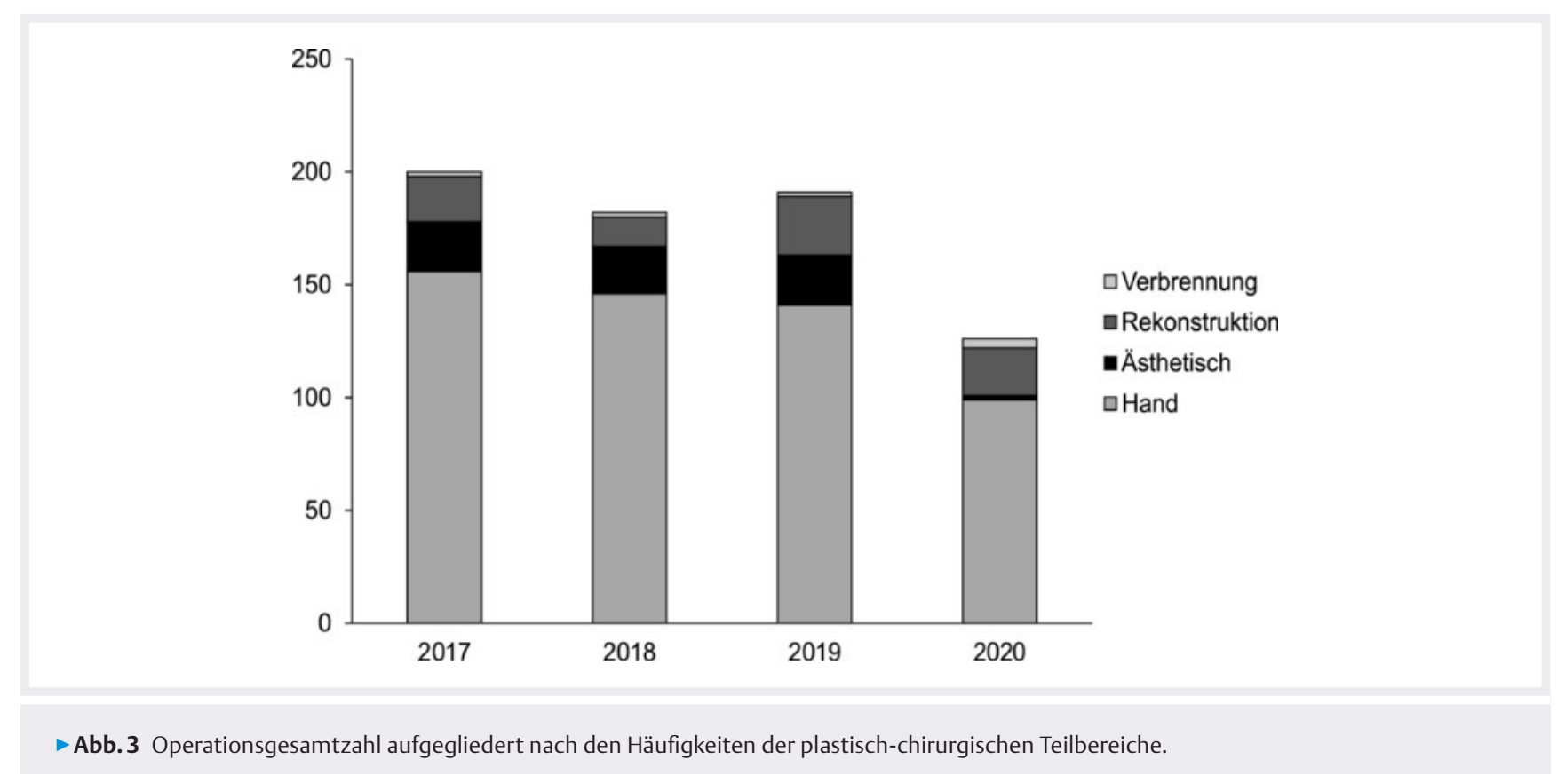

dringliche und 68,3 elektive. Während der SARS-CoV-2-Restriktionen und der begleitenden Reduktion auf notfallmäßige und dringliche Operationen erfolgten 110 Operationen, was ca. 57,3\% der durchschnittlich durchgeführten Operationen in den Jahren zuvor entsprach. Die Gesamtzahl an OPs 2020 setzt sich auf Grund der Restriktionen ausschließlich aus Notfällen und dringlichen OPs zusammen. Dem entsprechend erfolgten 62 notfallmäßige und 48 dringliche OPs. Um die Vergleichbarkeit zu waren, wurde für die Untersuchung der Unterschiede der Häufigkeiten unter dem Gesichtspunkt der OP-Dringlichkeit auch von den Jahren 2017-2019 die OP-Gesamtzahl ohne die elektiven Operationen geführt. Dies entsprach einer durchschnittlichen OP-Anzahl von 120,7. Der prozentuale Anteil der notfallmäßig durchgeführten OPs zur Gesamtanzahl an Operationen liegt in den Jahren 2017-2019 bei 54,9\% und im Jahr 2020 bei 56,4\%, der von den dringlich durchgeführten OPs bei $45 \%$ in den Jahren 2017-2019 und 44,6 \% im Jahre 2020 ( $\bullet$ Abb. 1).

Von den 192 Operationen der Jahre 2017-2019 waren durchschnittlich 32 (16,7\%) berufsgenossenschaftlich versicherte Fälle. Betrachtet man auch hier ausschließlich der notfallmäßigen und dringlichen Operationen beträgt die Anzahl der berufsgenossenschaftlich versicherten Fälle 27 (22,4\%). Während der SARS-CoV-2Restriktionen fanden 22 Operationen im Rahmen von berufsgenossenschaftlich versicherten Fällen statt. Dies entsprach einem Anteil von $20 \%$ der Gesamtanzahl an Operationen ( $\triangleright$ Abb. 2 ).

Schlüsselt man die erfolgten Operationen nach den plastischchirurgischen Teilbereichen auf, zeigt sich die deutliche Mehrheit der handchirurgischen Eingriffe ( $\bullet$ Abb. 3 und $\triangleright$ Abb. 4 ). Der Anteil notfallmäßiger handchirurgischer Eingriffe zeigte im Gegensatz zu den elektiven Eingriffen keine deutliche Veränderung während der SARS-CoV-2-Restriktionen. Ähnliches gilt für die dringlichen handchirurgischen Eingriffe.

Der geforderte Stopp der elektiven und vor allem ästhetischen Operationen während der SARS-CoV-2-Restriktionen wurde umgesetzt.
Die Unfallursache bei notfallmäßigen und dringlichen handchirurgischen Eingriffen während der SARS-CoV-2-Restriktionen im Vergleich zu den Vorjahren zeigte keine relevanten Unterschiede während der Restriktionen ( $\triangleright$ Abb. 5 und $\triangleright$ Abb. 6). Schnittverletzungen, Infekte und Sägeverletzungen bildeten dabei die Hauptursachen. Bei den dringlich durchgeführten Eingriffen zeigte sich eine tendenzielle Reduktion der Sportverletzungen und Sekundäreingriffe während der SARS-CoV-2-Restriktionen.

\section{Diskussion}

Im Rahmen der SARS-CoV-2-Pandemie kam das elektive Programm am beobachteten Zentrum vollständig zum Erliegen. Trotz der Einschränkungen des täglichen Lebens für weite Teile der Bevölkerung zeigte sich dennoch eine OP-Auslastung von 57,3\% im Vergleich zu den Vorjahren. Vor allem notfallmäßig durchgeführte Operationen ließen in ihrer absoluten Anzahl keinen deutlichen Unterschied zwischen der Zeit der SARS-CoV-2-Restriktionen und den vergangenen Jahren erkennen. Ähnliche Daten konnten durch die Kollegen aus Graz gezeigt werden. „Rund 50 \% der Normal-OP-Kapazität“ bedurfte es auch da für notfallmäßige Eingriffe [13]. Im Gegensatz zu den Daten aus Chicago standen, wie in den Jahren zuvor, die handchirurgischen Notfälle im Vordergrund. Anders als Hassan et al. kam es nicht zu einem deutlichen Rückgang an unfallbedingten Handverletzungen [14]. Daher ergibt sich aus den vorliegenden Daten eine eindeutige Erforderlichkeit der Vorhaltung von handchirurgischen OP-Kapazitäten und Infrastrukturen auch während Krisenzeiten.

Ähnliches gilt für die dringlich durchzuführenden Eingriffe. Rekonstruktionen von Defekten durch Traumata, Infektionen oder Tumore sowie deren potentielle Folgeeingriffe bildeten auch während der Krise die Hauptindikation und sind nicht zu verschieben, solange die Individualmedizin nicht zugunsten einer Katastrophenmedizin abgelöst wird. 

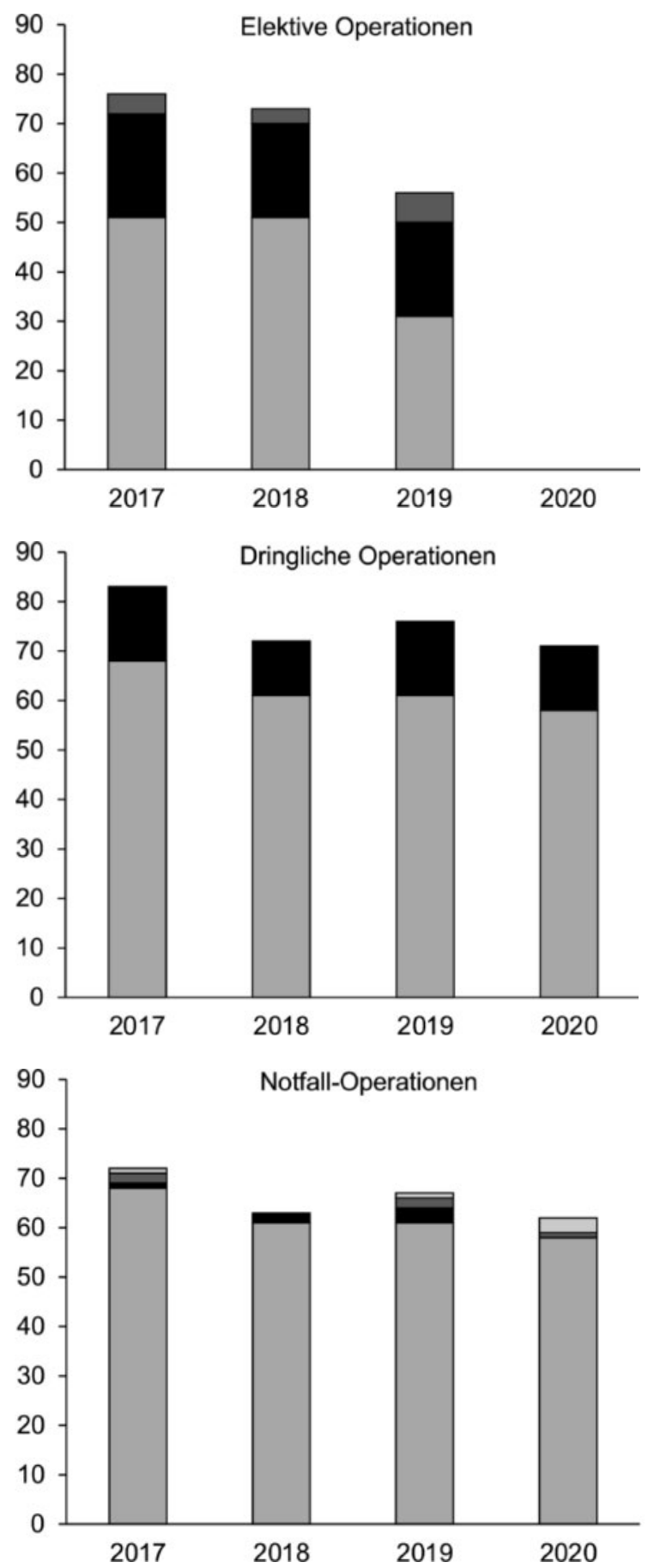

$\square$ Verbrennung

aRekonstruktion

ästhetisch

口Hand

- Abb. 4 Häufigkeitsanteile der Operationen bezogen auf die Operationsdringlichkeit.

Die Anzahl an berufsgenossenschaftlich versicherten Fällen ergab keine bewertbare Veränderung zu den Vorjahren. Die Ursache hierfür liegt am ehesten in der fortgesetzten Arbeitstätigkeit in Bereichen, die kein Home-Office erlauben. Da häufig in diesen Bereichen auch keine Kurzarbeit erfolgte, ist das Unfallaufkommen erklärbar. Es ist anzunehmen, dass in Fällen eines Lockdowns, wie beispielsweise in Italien oder Spanien, andere Ergebnisse zu erwarten wären.
Die vorliegenden Daten zeigen einen höheren Anteil an Notfalloperationen durch nicht berufsgenossenschaftlich versicherte Unfälle. Die genaue Ursache lässt sich anhand der vorliegenden Daten nur vermuten. Es ist anzunehmen, dass mehr handwerkliche Tätigkeiten im Heim- und Gartenbereich durch Kurzarbeit, Home-Office und der Beschränkung der Reisefreiheit während der Osterferien durchgeführt wurden. 

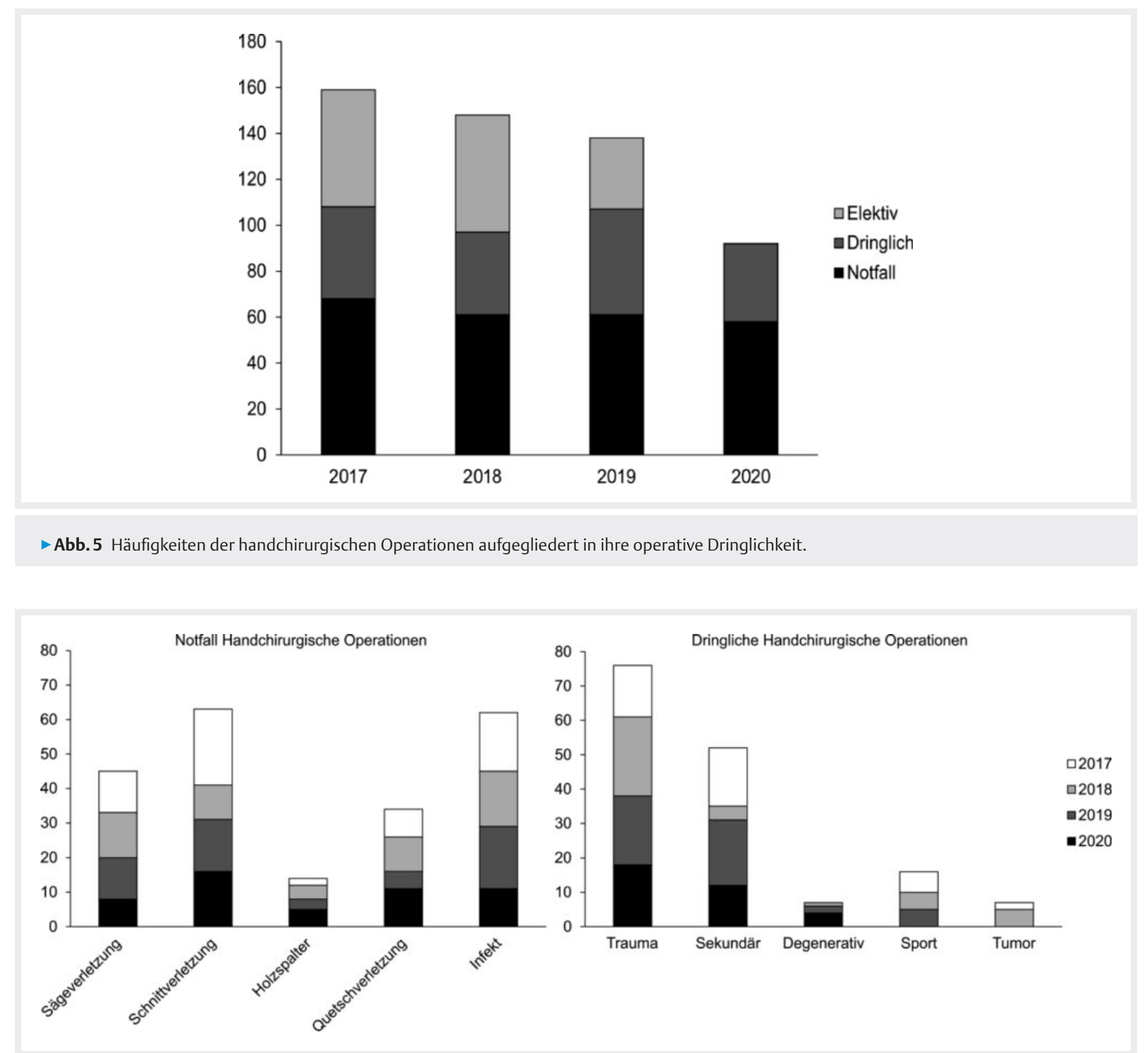

Abb. 6 Ursachen handchirurgischer Operationen aufgegliedert nach der Häufigkeit in den letzten Jahren.

Zusammenfassend zeigt sich, dass die plastisch-chirurgische Versorgung in einer überwiegend rekonstruktiv ausgerichteten Großklinik während der SARS-CoV-2-Pandemie zwar deutlich eingeschränkt verfügbar war, dennoch aber auf Grund der Notfallversorgung systemrelevant bleibt.

Das Fachgebiet der Plastisch-Ästhetischen Chirurgie ist traditionell sehr breit und beinhaltet alle 4 Säulen einschließlich der Handchirurgie. Daher ist aufgrund der jeweils an die örtliche Infrastruktur, Patientenaufkommen und auch gewachsene Strukturen adaptierten Ausrichtung der Plastischen Chirurgie ein direkter Vergleich einzelner Kliniken oft schwierig. Hierzu wurde bereits von anderen Autoren - etwa in Bezug auf die Forschungsleistung und auf die interdisziplinäre Zusammenarbeit - ein Zusammenhang zwischen der Organisationsstruktur von plastisch-chirurgischen Abteilungen/Kliniken/Sektionen an Universitätsklinika be- schrieben $[15,16]$. In einem der beteiligten Zentren wurden auch während der kritischen Phase im interdisziplinären Konsensus Operationen an der weiblichen Brust vorgenommen, die etwa der Beseitigung von schmerzhaften Kapselfibrosen dienten. Lediglich planbare autologe Brustrekonstruktionen und ästhetisch bedingte formverändernde Eingriffe unterschiedlicher Art wurden konsequent verschoben. Besonders in der Anfangsphase der PandemieBeschränkungen zeigte sich, dass eine anfangs tägliche offene und schonungslose interdisziplinäre Diskussion der jeweiligen Operationspläne im Kreis der Verantwortlichen auch in anderen chirurgischen Fachgebieten zu einer restriktiven Indikationsstellung beitragen kann. Medizinisch notwendige Eingriffe des Plastisch-Chirurgischen Fachgebietes konnten in diesen kritischen Diskussionen ebenso ihren Stellenwert belegen, wie vergleichbare Dringlichkeiten anderer Disziplinen. 
- Tab. 1 Evaluierte Daten der OP-Auslastung im Zeitraum 16.03.2017-2020.

\begin{tabular}{|c|c|c|c|c|c|c|}
\hline & & & 2017 & 2018 & 2019 & 2020 \\
\hline \multirow[t]{3}{*}{ Patienten } & & & 187 & 175 & 136 & 110 \\
\hline & männlich & & 123 & 117 & 85 & 83 \\
\hline & weiblich & & 64 & 58 & 51 & 27 \\
\hline \multirow[t]{12}{*}{ OPs gesamt } & & & 203 & 185 & 187 & 110 \\
\hline & BG-lich & & 42 & 33 & 21 & 22 \\
\hline & nicht BG-lich & & 161 & 152 & 166 & 88 \\
\hline & ambulant & & 79 & 66 & 72 & 48 \\
\hline & stationär & & 124 & 119 & 115 & 71 \\
\hline & ästhetisch & & 22 & 21 & 22 & 2 \\
\hline & Rekonstruktion & & 20 & 13 & 26 & 21 \\
\hline & Verbrennung & & 2 & 2 & 2 & 4 \\
\hline & Hand & & 156 & 146 & 141 & 99 \\
\hline & Notfälle & & 70 & 62 & 67 & 62 \\
\hline & elektiv & & 76 & 73 & 56 & 0 \\
\hline & dringlich & & 57 & 50 & 56 & 48 \\
\hline \multirow[t]{9}{*}{ Notfall } & ästhetisch & & 1 & 2 & 3 & 0 \\
\hline & Rekonstruktion & & 2 & 0 & 2 & 1 \\
\hline & Verbrennung & & 1 & 0 & 1 & 3 \\
\hline & Hand & & 68 & 61 & 61 & 58 \\
\hline & & Sägeverletzung & 12 & 13 & 12 & 8 \\
\hline & & Schnittverletzung & 22 & 10 & 15 & 16 \\
\hline & & Holzspalter & 2 & 4 & 3 & 5 \\
\hline & & Quetschverletzung & 8 & 10 & 5 & 11 \\
\hline & & Infekt & 17 & 16 & 18 & 11 \\
\hline \multirow[t]{6}{*}{ Elektiv } & ästhetisch & & 21 & 19 & 19 & 0 \\
\hline & Rekonstruktion & & 4 & 3 & 6 & 0 \\
\hline & Hand & & 51 & 51 & 31 & 0 \\
\hline & & sekundär & 13 & 14 & 8 & 0 \\
\hline & & Fehlbildung & 0 & 1 & 1 & 0 \\
\hline & & degenerativ & 38 & 36 & 22 & 0 \\
\hline \multirow[t]{12}{*}{ Dringlich } & Tumor & & 2 & 3 & 3 & 1 \\
\hline & Rekonstruktion & & 15 & 11 & 15 & 13 \\
\hline & & Verbrennung & 2 & 2 & 1 & 1 \\
\hline & & Trauma & 5 & 3 & 8 & 7 \\
\hline & & Tumor & 3 & 4 & 4 & 1 \\
\hline & & postop WHS & 5 & 2 & 2 & 4 \\
\hline & Hand & & 40 & 36 & 46 & 34 \\
\hline & & Trauma & 15 & 23 & 20 & 18 \\
\hline & & sekundär & 17 & 4 & 19 & 12 \\
\hline & & degenerativ & 0 & 1 & 2 & 4 \\
\hline & & Sport & 6 & 5 & 5 & 0 \\
\hline & & Tumor & 2 & 5 & 0 & 0 \\
\hline
\end{tabular}

Demnach scheint die in Großkliniken erforderliche Fokussierung auf die Säulen Rekonstruktion, Handchirurgie und Verbrennungsbehandlung, im Gegensatz zu mehr ästhetisch-plastisch ausgerichteten Institutionen, die unmittelbaren Auswirkungen der Pandemie auf das operative Alltagsgeschehen abzumildern. Gleichzeitig kann aber auch die Relevanz dieses Fachgebiets für die allgemeine Patientenversorgung belegt werden. Anders als in der allgemeinen Wahrnehmung, ggf. auch innerhalb eines Großklinikums, leistet die Plastische Chirurgie einen sehr wichtigen Anteil der Grundversorgung. Vor dem Hintergrund, dass unsere Disziplin nachweislich, be- 
ginnend mit der Beschreibung der indischen Nasenersatzplastik vor über 2500 Jahren, mit bahnbrechenden innovativen Ansätze zum medizinischen Fortschritt beiträgt, sind die Autoren der Ansicht, dass auch der Einsatz in Krisenzeiten zur weiteren Konsolidierung unserer Disziplin beitragen kann.

Interessenkonflikt

Die Autorinnen/Autoren geben an, dass kein Interessenkonflikt besteht.

\section{Literaturverzeichnis}

[1] Remuzzi A, Remuzzi G. COVID-19 and Italy: what next? Lancet 2020; 395: 1225-1228. Im Internet: https://linkinghub.elsevier.com/retrieve/pii/S0140673620306279

[2] Saglietto A, D'Ascenzo F, Zoccai GB et al. COVID-19 in Europe: the Italian lesson. Lancet 2020; 395: 1110-1111. DOI:10.1016/S01406736(20)30690-5

[3] Goyal P, Choi J], Pinheiro LC et al. Clinical Characteristics of Covid-19 in New York City. N Engl J Med 2020; 382: 1-3. Im Internet: DOI:10.1056/NEJMc2005203

[4] Culp WC. Coronavirus Disease 2019. A A Pract 2020; 14: e01218. Im Internet: http://journals.Iww.com/10.1213/XAA.0000000000001218

[5] Yang X, Yu Y, Xu J et al. Clinical course and outcomes of critically ill patients with SARS-CoV-2 pneumonia in Wuhan, China: a single-centered, retrospective, observational study. Lancet Respir Med 2020; 8: 475-481

[6] Grasselli G, Pesenti A, Cecconi M. Critical Care Utilization for the COVID-19 Outbreak in Lombardy, Italy: Early Experience and Forecast during an Emergency Response. J Am Med Assoc 2020; 323 : 1545-1546

[7] Xie J, Tong Z, Guan X et al. Critical care crisis and some recommendations during the COVID-19 epidemic in China. Intensive Care Med 2020; 46: 837-840. DOI:10.1007/s00134-020-05979-7

[8] Giunta RE, Frank K, Costa $\mathrm{H}$ et al. The COVID-19 Pandemic and its Impact on Plastic Surgery in Europe - An ESPRAS Survey. Handchir Mikrochir Plast Chir 2020; 52: 221-232

[9] van Heijningen I, Frank K, Almeida F et al. EASAPS/ESPRAS Considerations in getting back to work in Plastic Surgery with the COVID-19 Pandemic - A European point of view. Handchir Mikrochir Plast Chir 2020. DOI:10.1055/a-1175-4169

[10] Bayerischen Staatsministeriums des Innern für S und I sowie des BS für $\mathrm{G}$ und P. Notfallplan Corona-Pandemie: Allgemeinverfügung zur Bewältigung erheblicher Patientenzahlen in Krankenhäusern Bekanntmachung. Bayer Minist 2020; 1-8

[11] American College of Surgeons. COVID-19: Guidance for Triage of Non-Emergent Surgical Procedures. Am Coll Surg 2020; 1-38. Im Internet: https://www.facs.org/COVID-19/clinical-guidance/triage\%0Ainternal-pdf://0.0.6.158/triage.html

[12] Giunta RE, Frank K, Moellhoff N et al. Die COVID-19 Pandemie und ihre Folgen für die Plastische Chirurgie und Handchirurgie. Handchir Mikrochir Plast Chir 2020. DOI:10.1055/a-1163-9009

[13] Kamolz LP, Spendel S. Die COVID-19 Pandemie und ihre Folgen für Plastische Chirurgie und Handchirurgie: ein Kommentar aus Grazer Sicht. Handchir Mikrochir Plast Chir 2020; 52: 242-243. DOI:10.1055/a-1163-9009

[14] Hassan K, Prescher H, Wang F et al. Evaluating the Effects of COVID-19 on Plastic Surgery Emergencies. Ann Plast Surg 2020; Publish Ah: 1-24
[15] Kemper R, Horch R, Giunta R et al. Interdisziplinarität in der akademischen Plastischen Chirurgie - belanglos oder zukunftsorientiert? Handchir Mikrochir Plast Chir 2019; 51: 144-150. DOI:10.1055/a-0795-3583

[16] Giunta RE, Möllhoff N, Horch RE et al. Register Forschungsförderung der Deutschen Gesellschaft der Plastischen, Rekonstruktiven und Ästhetischen Chirurgen (DGPRÄC) und Forschungsförderungs-Bericht 2017/2018. Handchir Mikrochir Plast Chir 2018; 50: 414-421. DOI:10.1055/s-0042-121417 